F)

\title{
Robotic-assisted THA can achieve precise cup positioning even in severe developmental dysplasia of hip
}

\author{
Shinya Hayashi ${ }^{1}$, Shingo Hashimoto ${ }^{1}$, Koji Takayama ${ }^{1}$, Tomoyuki Matsumoto ${ }^{1}$, \\ Nao Shibanuma ${ }^{2}$, Kazunari Ishida ${ }^{2}$, Ryosuke Kuroda ${ }^{1}$ \\ 1. Department of Orthopaedic Surgery, Kobe University Graduate School of Medicine
}

2. Kobe Kaisei Hospital

S11793290@yahoo.co.jp

\section{Introduction}

Acetabular cup malposition is recognized as a risk factor for dislocation and increased wear after total hip arthroplasty (THA) (1). However, it was difficult to achieve target cup positioning even by experienced surgeons (1). Especially, developmental hip dysplasia (DDH) presents a considerable difficult for precise cup placement in THA. Several papers demonstrated the accuracy of cup placement of DDH patients by using computed tomography (CT)-based navigation (2), but no report was found by using robotic-assisted THA. Recently, new generation of robotic-assisted total hip arthroplasty (THA) was introduced and precise cup positioning was achieved during THA (3)

We hypothesize robotic-assisted THA can achieve precise cup positioning. The aim of this study is to figure out the accuracy of cup placement of severe DDH cases by using robotic-assisted THA

\section{Methods}

This was a prospective cohort study. The study analyzed a consecutive series of 53 hips with roboticassisted THA between Aug 2018 and Sep 2019. Fifteen patients were DDH cases, and classified Crowe type II- 7 patients, type III- 7 patients, and type IV- 1 patient. The mean age of the subjects was 63.2 years and the average body mass index (BMI) was $24.1 \pm 5.4 \mathrm{~kg} / \mathrm{m}^{2}$. All patients underwent robotic assisted-THA (MAKO Rio Robot, Ft. Lauderdale, FL) for osteoarthritis via the Hardinge or posterior approach in the lateral position. The patients underwent THA using Trident hemispherical cup and Accolade II or Exeter v40 stems (Stryker, Kalamazoo, MI). Postoperatively, computer-aided design models of the implants were manually adjusted for postoperative multi-planar reconstruction of CT images using 3D template software (ZedHip, Lexi, Japan). Cup inclination and anteversion angles were measured with respect to the functional pelvic plane. To analyze the accuracy of intraoperative navigation records for cup inclination and anteversion, we compared the intraoperative cup angles using the navigation records with the postoperative angles using postoperative CT data. 
The differences between intraoperative cup angles using navigation records or preoperative target angles and postoperative angles using postoperative CT data were measured, and the absolute difference was compared using the Mann-Whitney U test.

\section{Results}

\section{Reproducibility of robotic-assisted THA for preoperative plan}

In non DDH cases, the average measurement absolute error (postoperative CT-preoperative target) was $1.9 \pm 2.1^{\circ}$ (inclination) and $2.0 \pm 2.5^{\circ}$ (anteversion) (Figure 1). In DDH cases, the average measurement absolute error (postoperative CT- preoperative target) was $2.2 \pm 3.1^{\circ}$ (inclination) and 2.6 $\pm 2.4^{\circ}$ (anteversion) (Figure 1). There were no differences of the average measurement absolute error between DDH and non DDH cases.

\section{Accuracy of navigation record for cup inclination and anteversion angles}

In non DDH cases, the average measurement absolute error (postoperative CT-navigation record) was $2.3 \pm 1.9^{\circ}$ (inclination) and $2.2 \pm 1.5^{\circ}$ (anteversion) (Figure 1). In DDH cases, the average measurement absolute error (postoperative CT-navigation record) was $2.0 \pm 2.5^{\circ}$ (inclination) and 2.3 $\pm 2.7^{\circ}$ (anteversion) (Figure 1). There were no differences of the average measurement absolute error between DDH and non DDH cases. Scatter plots demonstrated the measurement error of both inclination and anteversion within $5^{\circ}$ was $89.5 \%$ in non DDH cases (Figure 2a) and $80 \%$ in DDH cases (Figure 2b).

\section{Discussion}

In this study, we demonstrated that no differences of the average measurement absolute navigation record error or the absolute difference between target angle and postoperative cup alignment were found between severe DDH and non-DDH cases. Our results indicate that this robotic-assisted system has high accuracy for cup placement even in severe DDH case.

Kanawade et al. reported the accuracy of same robotic-assisted system for non DDH cases (4). Postoperative CT validation of the intraoperative inclination record within $5^{\circ}$ was $88 \%$ and anteversion record was $84 \%$ (4). Kamara et al. also reported the accuracy that $90 \%$ of patients were placed the cup in Lewinnek safe zone (3). These results were similar as our evaluation.

In conclusion, robotic-assisted THA can achieve precise cup positioning even in severe developmental hip dysplasia.

\section{Figure 1}

\begin{tabular}{|l|l|l|l|l|l|l|}
\hline & \multicolumn{2}{|c|}{ non DDH } & & \multicolumn{2}{c|}{ DDH } & \\
\hline & $\begin{array}{l}\text { Radiographic } \\
\text { inclination }\end{array}$ & $\begin{array}{l}\text { Radiographic } \\
\text { anteversion }\end{array}$ & P-value & $\begin{array}{l}\text { Radiographic } \\
\text { inclination }\end{array}$ & $\begin{array}{l}\text { Radiographic } \\
\text { anteversion }\end{array}$ & P-value \\
\hline $\begin{array}{l}\text { Absolute } \\
\text { difference } \\
\text { (postoperative- } \\
\text { navigation } \\
\text { records }\end{array}$ & $1.9 \pm 2.1^{\circ}$ & $2.0 \pm 2.5^{\circ}$ & 0.937 & $2.2 \pm 3.1^{\circ}$ & $2.6 \pm 2.4^{\circ}$ & 0.11 \\
\hline $\begin{array}{l}\text { Absolute } \\
\text { difference } \\
\text { (postoperative- } \\
\text { target angle) }\end{array}$ & $2.3 \pm 1.9^{\circ}$ & $2.2 \pm 1.5^{\circ}$ & 0.347 & $2.0 \pm 2.5^{\circ}$ & $2.3 \pm 2.7^{\circ}$ & 0.386 \\
\hline
\end{tabular}




\section{Figure 2}
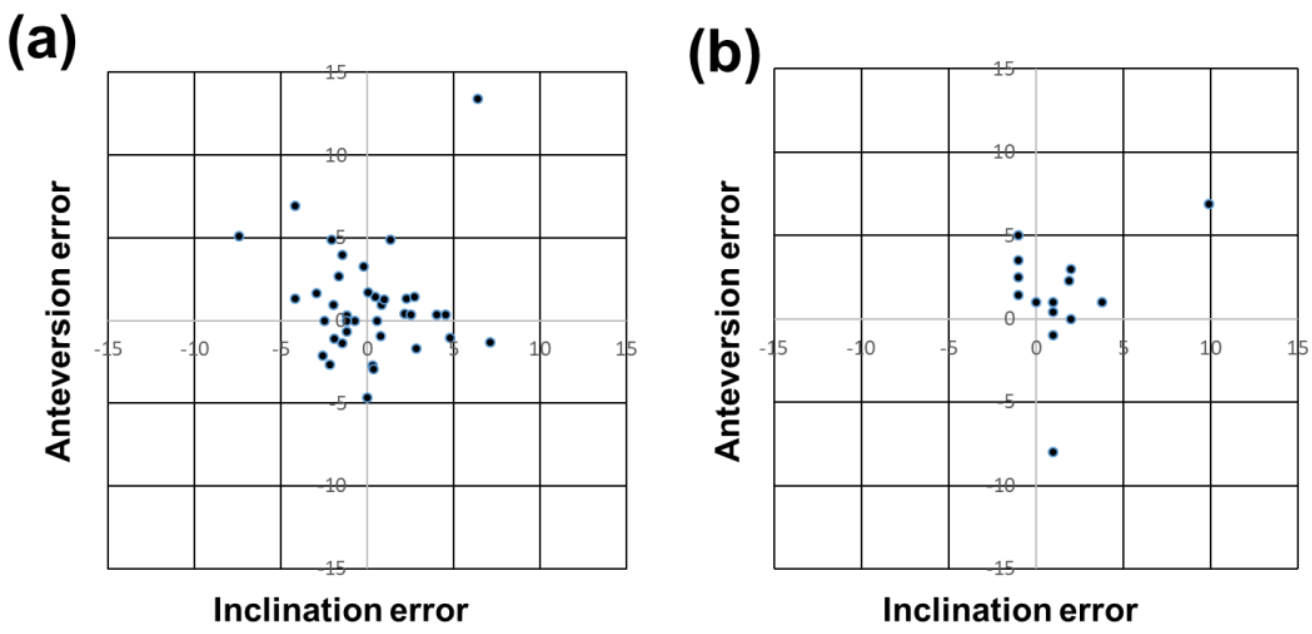

\section{Figure legend}

Figure 1 Results of cup orientation

Figure 2 Scatter plot of the absolute difference in radiographic inclination and anteversion between postoperative computed tomography (CT) analysis and intraoperative navigation records.

\section{References}

1. Lewinnek GE, Lewis JL, Tarr R, Compere CL, Zimmerman JR. Dislocations after total hipreplacement arthroplasties. J Bone Joint Surg Am1978 Mar;60(2):217-20.

2. Yamada K, Endo H, Tetsunaga T, Miyake T, Sanki T, Ozaki T. Accuracy of Cup Positioning With the Computed Tomography-Based Two-dimensional to Three-Dimensional Matched Navigation System: A Prospective, Randomized Controlled Study. J Arthroplasty2018 Jan;33(1):136-43.

3. Kamara E, Robinson J, Bas MA, Rodriguez JA, Hepinstall MS. Adoption of Robotic vs Fluoroscopic Guidance in Total Hip Arthroplasty: Is Acetabular Positioning Improved in the Learning Curve? J Arthroplasty2017 Jan;32(1):125-30.

4. Kanawade V, Dorr LD, Banks SA, Zhang Z, Wan Z. Precision of robotic guided instrumentation for acetabular component positioning. J Arthroplasty2015 Mar;30(3):392-7. 